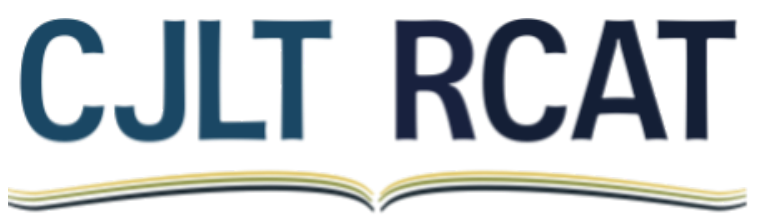

Canadian Journal of Learning and Technology

La Revue canadienne de l'apprentissage et de la technologie

Volume 47 (4)

Special Issue 2021

\title{
Approches et paradigmes pour la recherche sur les usages éducatifs des technologies. Enjeux et perspectives
}

\section{Approaches and Paradigms for Research on the Educational uses of Technologies: Challenges and Perspectives}

Georges-Louis Baron, Université de Paris, Laboratoire EDA

Cedric Fluckiger, Université de Lille, Laboratoire CIREL

\section{Résumé}

Les lignes qui suivent présentent une réflexion commencée à l'EDUsummIT 2019 à Québec, notamment dans un groupe de travail sur les paradigmes de recherche souhaitables dans le domaine de l'étude des usages éducatifs des technologies de l'information et de la communication. Il s'agit de déconstruire la notion de passage à l'échelle des innovations et interroge la tension entre différents paradigmes de recherche, critique les points de vue peu problématisés de certains décideurs qui promeuvent un type particulier de recherche se concentrant uniquement sur le passage à l'échelle uniquement en matière de résultats. Finalement, l'article argumente en faveur de l'intérêt d'approches participatives plurielles associant des collectifs hybrides durables.

Mots clés : recherche coopérative ; diffusion des innovations ; paradigme ; numérique éducatif

\begin{abstract}
The following lines present a reflection started at the EDUsummIT2019 in Quebec City, notably in a working group on desirable research paradigms in the study of educational uses of information and communication technologies. It deconstructs the notion of scaling up innovations and questions the tension between different research paradigms, criticizing the simplistic views of some policy makers who promote a particular type of research that focuses solely on scaling up in terms of results. Finally, the paper argues for plural participatory approaches that associate sustainable hybrid collectives.
\end{abstract}

Keywords: Collaborative research; Diffusion of innovations; Paradigm; Digital education 


\section{Éléments d'analyse d'un contexte troublé}

\section{Technologies de l'information et de la communication au temps de la COVID-19}

Les perturbations dramatiques entrainées par la crise sanitaire du coronavirus ont eu pour effet d'infléchir, peut-être durablement, la place de la formation à distance et des approches pédagogiques fondées sur le recours à des ressources en ligne.

À la base une question préoccupant surtout chercheurs et institutions, la mise à distance des enseignements est devenue un problème social, discuté dans la presse et dans les familles. Désormais, l'usage des technologies est pratiquement incontournable en éducation, créant un contexte interrogeant les approches pédagogiques classiques.

Face à cette évolution, les attentes sociales, médiatiques et institutionnelles vis-à-vis de la recherche risquent d'être modifiées dans le sens d'une demande plus forte de production d'un discours prescriptif, sur ce qu'il convient de faire pour faciliter les conditions nouvelles de l'exercice de l'enseignement.

Un exemple en a récemment été donné, en France, par le Conseil scientifique de l'Éducation nationale (CSEN). Ce dernier se promettait, en mai 2020, de publier prochainement « des recommandations sur les types de recherche translationnelle qui ont leur place en éducation, les différents niveaux de preuve qu'ils apportent, et leurs enjeux éthiques et pratiques ${ }^{1} »$, afin d'indiquer aux enseignants les ressources ou logiciels « qui ont, au moins en partie, fait leurs preuves » ou « ayant démontré leur efficacité ». Cette demande, facilement compréhensible, trouve un écho chez les décideurs politiques locaux devant investir dans le domaine éducatif. Elle peut être relayée par des parents et des praticiens trouvant normal que la recherche indique «ce qui marche $»^{2}$.

On voit périodiquement les limites d'une gouvernance revendiquant un pilotage par les résultats de la science, ou plus exactement par des experts. Ainsi, au début de la pandémie du coronavirus, l'expertise était très fragile, car on était face à un agent infectieux nouveau et il n'y avait pas de consensus clairement établi. Par ailleurs, en matière de décisions politiques, les raisons scientifiques ont une importance relative, comme il est maintenant bien connu depuis Max Weber et son essai sur le savant et le politique (1919/1963).

Les circonstances actuelles appellent une nouvelle réflexion sur la spécificité des apports des chercheurs aux débats publics dans les domaines sur lesquels portent leurs recherches. Nous allons l'ébaucher ici en discutant les tensions, dans le domaine de la technologie éducative, entre différents paradigmes idéal typiques. Nous commencerons par analyser une notion centrale, celle du passage à l'échelle de la recherche et des innovations.

\footnotetext{
${ }^{1}$ Conseil scientifique de l'Éducation nationale (CSEN). Repéré à https://www.education.gouv.fr/csen-recommandationspedagogiques-COVID19

${ }^{2}$ https://www.education.gouv.fr/csen-recommandations-pedagogiques-COVID19
} 


\section{Une nécessaire rupture avec le sens commun : à propos de passage à l'échelle de la recherche et des innovations}

On sait depuis Bachelard que « le progrès scientifique manifeste toujours une rupture [...] entre connaissance commune et connaissance scientifique » (Bachelard, 1953, p. 207). Si le monde était ce qu'il paraît être, il n'y aurait pas besoin de faire de science. Il en est ainsi de l'objet même de cet article, la technologie éducative. Dans l'imaginaire collectif, dans bien des discours institutionnels (Fluckiger, 20120a), l'innovation pédagogique est constitutive de la technologie éducative, alors même que les recherches ont montré (Bernard \& Fluckiger, 2019) que ce n'est pas le cas. Il en est de même avec les représentations de sens commun sur la manière dont les innovations peuvent se diffuser plus largement.

C'est pourquoi il est indispensable de contribuer à clarifier les termes du débat. En effet, le problème qui occupe les décideurs actuellement n'est pas tant celui de l'innovation technologique en tant que telle, mais plutôt l'innovation institutionnelle, dans le sens que Norbert Alter (2000) donnait à ce terme, qui est associé au souhait de «passage à l'échelle».

De très nombreuses recherches ont été consacrées à cette question. En 2003, Coburn observait qu'il est nécessaire de considérer autre chose que le nombre d'écoles concernées et de prendre aussi en compte la profondeur des innovations, leur durabilité, leur diffusion et les changements dans l'appropriation de l'innovation pour de nouveaux acteurs.

Pour Morel et ses collègues (2019), la notion est multiple et ne génère pas les mêmes problématiques : ces auteurs considèrent le passage à l'échelle comme un processus dynamique, avec plusieurs étapes : la simple adoption, la réplication, l'adaptation, la réinvention.

Par exemple l'adoption généralisée du courrier électronique (et plus largement de la communication écrite par Internet) s'est bel et bien produite en une dizaine d'années, depuis la fin des années 1980. Elle a eu des effets collatéraux non négligeables dans les manières de communiquer.

La réplication, souhaitée en général par les décideurs, peut être celle des résultats, dont on attend qu'ils restent les mêmes dans des contextes différents, ou bien celle des procédures elles-mêmes. La vue du passage à l'échelle par adaptation rejette la notion de stricte fidélité à l'innovation princeps, en considérant que les adaptations locales vont conduire à de meilleurs résultats. Cependant, le problème des limites à ne pas dépasser pour que cette adaptation ne dévie pas trop des principes de base est posé. La réinvention, pour sa part, correspond à une intention délibérée de s'appuyer sur les précédentes innovations, qui servent de catalyseurs pour aller plus loin et correspond à une sorte de remixage, comme le promeut la culture du logiciel ou des ressources libres.

Cette dernière variété du passage à l'échelle est proche de celle proposée par Martinand dès 2002. Pour lui, la notion populaire de transfert de connaissances ne rend pas compte de la réalité. Il propose à la place trois métaphores : la circulation (des savoirs, des textes, de l'information), qui peut conduire à des détournements; la traduction, au sens de la sociologie de la traduction promue par 
Michel Callon et, finalement, la production de connaissances dans des processus d'action, c'est-à-dire la recherche-intervention, « ni science appliquée ni action militante » qui est essentiellement menée en partenariat, dans des conditions qui s'opposent aussi bien à celles de la recherche de laboratoire, qui, elle, réalise un « confinement de l'action », qu'au modèle du terrain, qui « naturalise les phénomènes « en masquant ce qui n'existe que par l'action » (p. 90).

Il souligne que l'enjeu est de parvenir à une reproblématisation des résultats obtenus, c'est-àdire, dans le meilleur des cas, une co-problématisation avec d'autres acteurs, praticiens et décideurs. Bien sûr, cela pose des problèmes dans un domaine où les chercheurs, qui ont déjà problématisé la question, sont en situation asymétrique par rapport aux autres acteurs.

Une des conséquences souvent attribuées au manque de passage à l'échelle comme réplication est l'impression que l'histoire se répète en matière d'utilisation d'instruments techniques en éducation et que finalement rien ne bouge. Mais cette appréciation est trompeuse car il finit par se produire des « scolarisations » d'innovations (Baron \& Bruillard, 2004). Le problème est que ces processus se produisent très lentement. Ils conduisent de plus à des situations totalement banalisées, qui appellent de nouvelles innovations. Il s'agit d'un enchaînement sans fin programmée, dépendant de l'action de communautés de pratique et de collectifs qui portent des idées bien au-delà de la durée de vie des individus.

C'est ce qu'ont bien montré les nombreuses recherches sur la généralisation des innovations. Lorsqu'elles sont le fait d'enseignants « pionniers » (Béziat, 2003) ou d' « innovateurs bricoleurs » (Villemonteix \& Khaneboubi, 2013), il s'agit surtout de jouer avec les règles, parfois les détourner, dans des «stratégies d'effraction ou de détournement menées par des enseignants pionniers contre les usages prévus et prescrits » (Mœglin, 2012, p. 13). La difficulté est de dépasser le cercle des innovateurs. Lorsque les innovations sont uniquement portées par l'institution scolaire, les usages ont du mal à se pérenniser : on observe parfois une sorte de " gourmandise technophile » des enseignants (Fluckiger et al., 2016) mais après une période d'enthousiasme et d'adhésion, les contraintes conduisent souvent à revoir les ambitions pédagogiques à la baisse (Nogry \& Sort, 2016).

Par-delà la question du passage à l'échelle, ce qui intéresse les décideurs est celle de l'effet des nouveaux environnements disponibles en éducation. Question redoutable, à laquelle de nombreux travaux ont été consacrés.

Dans une synthèse récente, Cox (2018) observe que la question est compliquée par le caractère très évolutif des technologies qui perfusent dans l'ensemble de la société, au-delà des systèmes scolaires bien contrôlés (p. 1107) et par le fait que ce que les élèves apprennent n'est pas exactement ce que les enseignants attendaient. Comment définir ce que les enfants apprennent en dehors de l'école au moment où la frontière entre apprentissages formels et non formels est des plus floue? (p. 1113). Elle relève trois considérations à prendre en compte par la recherche : la perte de contrôle de ce que les apprenants utilisent en fait comme technologies; les évolutions des modalités sensorielles de 
l'apprentissage; la culture numérique des élèves habitués à de nouvelles formes de représentations (p. 1114).

Pour leur part, Baron et Depover (2019), dans un ouvrage s'intéressant aux effets du numérique en éducation, ont insisté sur l'aspect systémique de la question et distingué cinq dimensions : les effets sur les systèmes éducatifs et les environnements scolaires, sur les curricula, sur les compétences maitrisées par les élèves, sur les modèles et méthodes pédagogiques, sur les fonctions cognitives et les comportements sociaux des élèves.

Un problème fondamental, insistant, est celui des types de preuve. Cukurova et Luckin (2018) en distinguent quatre, qui gagnent à se conjuguer : preuves anecdotiques, descriptives, corrélationnelles et causales, auxquelles ils rajoutent un « méta-niveau » faisant appel à des méta-analyses confrontant les études existantes. Ces dernières, popularisées en éducation par Gene Glass depuis le milieu des années 1970, supposent cependant des recherches quantitatives suivant des modèles similaires (le « gold standard » étant l'expérimentation randomisée comparant les résultats de différents groupes) et permettant, notamment, de comparer des « tailles d'effets».

\section{Une forte pression institutionnelle en faveur des paradigmes expérimentaux}

Les approches promouvant la recherche expérimentale ont la faveur du milieu des décideurs, qui estiment avoir besoin de mesures permettant de juger du résultat des innovations et de guider leur action. On constate même depuis quelques années une forte pression pour préconiser, voire imposer, des modalités spécifiques de recherches expérimentales, sur le modèle de l'evidence based education (Saussez \& Lessard, 2009). Ce type de position avait fait l'objet d'une promotion active il y a déjà une vingtaine d'années aux É.-U. lors de la passation de la loi No child left behind, sous l'administration de G.W. Bush qui a affirmé sa volonté de fonder la décision sur des résultats scientifiques.

Comme le rappellent Saussez et Lessard (2009), il faut inscrire « le développement de l'approche de l' $^{\prime} \mathrm{BBP}^{3}$ aux États-Unis dans la perspective des changements introduits dans les politiques évaluatives au niveau fédéral à la fin des années 1970. Il se manifeste alors un intérêt pour les essais contrôlés randomisés » (p. 123). La pression institutionnelle pour ce type d'approches se répercute sur les choix de financement de la recherche.

Parmi les critiques faites à ce type d'approche promouvant une norme intangible (l'expérimentation randomisée avec groupe témoin), on peut citer celle de Pogrow (2017), qui se focalise sur des problèmes méthodologiques. Il en relève plusieurs qui expliquent d'après lui le manque de réplicabilité des résultats exposés dans des articles de recherche. Tout d'abord, il relève le fait que ce genre d'approche ne permet que de procéder à des comparaisons et ne donne pas d'indication sur des effets absolus, ce qui est d'un intérêt limité. Ensuite, il met en évidence que les résultats sont des

\footnotetext{
${ }^{3}$ Evidence based policy.
} 
comparaisons de scores ajustés en fonction de règles permettant de comparer des groupes hétérogènes (c'est aussi le cas avec le PISA, qui « normalise » les résultats obtenus).

Or, ces scores ajustés ne disent rien sur les résultats réels. Enfin, les « tailles d'effet» couramment calculées sont souvent trop faibles pour correspondre à des changements observables. De plus, elles font souvent l'objet de ce qu'il appelle des extrapolations hypothétiques, du genre : la méthode $M$ permet un gain de $X$ jours pour apprendre le sujet $Y$, qu'il est impossible de vérifier en pratique et qui agissent plutôt comme des arguments d'autorité. Il conclut que la pire des choses est de certifier comme efficaces des pratiques qui ne le sont pas et plaide pour des méthodes plus simples étudiant des effets visibles par les praticiens en s'assurant que les améliorations constatées sont consistantes d'une étude à l'autre.

Par ailleurs, Bart et Daunay (2016) se sont livrés à une analyse serrée des discours produits par le programme PISA. Outre l'absence de définition des termes désignant ce que PISA se propose de mesurer (les « compétences », « l'intelligence », etc.) ou encore la prétention assumée d'aller au-delà de la mesure (PISA affirme déterminer les « compétences qui leur serviront dans leur vie ultérieure », voir Bart et Daunay, 2016, p. 25), ils notent une rupture avec la pratique ordinaire des écrits scientifiques. Ainsi, « malgré l'importance et la récurrence de quelques-uns de ces travaux critiques et malgré la vivacité des polémiques que certains ont pu générer », on ne trouve pas de « débat argumenté avec les positions ou les thèses défendues par ces auteurs critiques, comme c'est le cas ordinairement dans les écrits scientifiques » (p. 22). Ils en concluent que « une fois gommées toutes les controverses, restent les choix faits effectivement, qui peuvent, sans contradictions, se parer des vertus de la certitude » (p. 23).

En somme, si les atours extérieurs de la scientificité sur le plan méthodologique sont présents, il manque ce qui est au fondement de la démarche scientifique : la définition et la construction conceptuelle des objets scientifiques étudiés (les « compétences », « l'intelligence », « l'innovation », les « outils numériques », etc.); la discussion des limites des approches méthodologiques choisies, qui sont implicitement supposées répondre à toute question; la discussion ouverte et la prise en compte des arguments opposés et des critiques (voir Fluckiger, 2020a, sur le cas du CSEN français).

\section{Deux grands paradigmes en tension pour les recherches sur les technologies en éducation}

Dans le domaine éducatif, et plus particulièrement dans celui de la technologie éducative, la question des paradigmes de recherche légitimes est ancienne. G. Mialaret, dans un article de synthèse sur la recherche en éducation (2016), rappelle l'importance historique de l'expérimentation dans sa constitution et le rôle important de la «pédagogie expérimentale » qui a des lettres de noblesse indéniables.

Il discute aussi d'autres approches légitimes, distinguant entre plusieurs types d'approche, précisant des méthodes associées, plaidant ainsi en fait pour le pluralisme et pour l'idée, logique, que 
ce qui compte c'est l'alignement des méthodes avec le type d'activité menée. Il relève finalement un problème, qui est en pratique redoutable :

Le problème fondamental, pour le chercheur, est donc de savoir faire lucidement la distinction entre ce qui relève de la « compréhension » et ce qui relève de « l'explication », pour donner aux deux séries de données leur propre statut épistémologique et ne pas tout mélanger dans le compte rendu de la recherche. (Mialaret, 2016, p. 68)

Différents types de réponse sont bien sûr apportés, en fonction des références théoriques des différentes communautés de recherche. Pour notre part, nous pensons qu'il est fructueux de distinguer, outre les approches proprement historiques, deux grandes familles de choix théoriques (que nous qualifierons de paradigmes) en tension dans les recherches actuelles sur la technologie éducative.

\section{Un paradigme applicationniste-prédictif}

On pourrait être tenté de qualifier ce premier paradigme d'applicationniste; mais c'est plutôt un paradigme de la prédiction. Il a notamment été porté il y a une vingtaine d'années aux É.-U. par la loi No child left behind, sur la base d'expérimentations contrôlées, qui doivent valider ou non l'efficacité d'outils ou de dispositifs, « l'accent étant mis sur les ressources éducatives et leur évaluation » (Bianco, $2018, \S 19)$. C'est en quelque sorte la transposition en éducation du « testé scientifiquement» pour les dentifrices. Cette conception est liée au courant de l'evidence based education (Saussez et applicationniste - prédictif Lessard, 2009) et engage à des études d'impact d'un outil ou d'une méthode, sur le modèle de ce qu'on connaît pour les médicaments. C'est elle qui est portée aujourd'hui par les instances étatiques ou leurs émanations, comme PISA (Bart \& Daunay, 2016).

Le problème est que cette approche peine à apporter des résultats fiables et généralisables (comme le note Bryk, 2017), précisément car le problème en éducation est bien celui du passage à l'échelle : les dynamiques de généralisation et de pérennisation des pratiques sont systémiques, et nécessitent des apports de toutes les sciences humaines et sociales pour bien les comprendre.

\section{Un paradigme compréhensif-explicatif}

On pourrait qualifier le second paradigme d'explicatif. Il ne vise pas vraiment à prédire l'efficacité d'un objet en supposant qu'il sera ensuite adopté sans problème dans les classes mais cherche à donner des éléments de compréhension et d'explication aux praticiens. Ce paradigme conduit à privilégier des études d'usages, à mettre au jour les difficultés effectives en situation de classe, à se confronter au point de vue des praticiens.

Cela conduit à mettre en question des mythes sur le numérique en éducation et à donner des faits et des outils de problématisation aux praticiens. Par exemple, le fait que l'introduction des technologies éducatives fonctionne selon des cycles d'illusion et de désillusion (Cuban, 1986) aide à prendre conscience de l'existence de discours doxiques souvent culpabilisants sur le numérique, la technologie éducative ou l'innovation pédagogique. Dans ce paradigme, les praticiens ne sont pas considérés comme des récipiendaires des résultats de la science, mais des partenaires. 
Un ensemble très important de travaux a porté sur les usages des technologies (Baron \& Bruillard, 1996; Jouet, 2000; voir aussi Fluckiger, 2020b pour un panorama récent des études des usages du numérique en éducation). Dans la mesure où les usages sont socialement partagés et même « pédagogiquement intégrés et institutionnellement validés » (Mœglin, 2012, p. 4), les recherches en relevant doivent caractériser et décrire les contextes sociaux, institutionnels, techniques qui rendent les pratiques intelligibles.

Ces orientations sont proches des approches dites critiques (De Munck, 2011; Selwyn, 2010; Collin et al., 2016). La mise au jour de formes de dominations, comme les discours institutionnels évoqués plus haut, permet en effet « la révélation des différentes formes de domination qui s'exercent dans le cadre d'un ordre social qui pèse sur le présent et qui, la plupart du temps, font l'objet d'une méconnaissance (c.-à-d. pas nécessairement d'une ignorance) de la part des sujets sociaux qui les subissent » (Granjon, 2014, p. 18-19).

La grande difficulté de ces approches est évidemment que le caractère contextualisé des observations : la prise en compte des spécificités saisies au niveau des acteurs, rend très difficile toute généralisation et aboutit le plus souvent à des résultats décevants du point de vue des décideurs. Plus largement, les questions auxquelles peuvent répondre ces approches sont limitées.

D'autres critiques lui sont adressées, comme celle de Bianco (2018), qui pose la question : « Peut-on fonder une science à partir de l'analyse de situations singulières? » (§15). Comment agréger les différents résultats? L'argument est sérieux, le risque d'une réduction de la recherche à un empirisme naïf, sans cumulativité des travaux, bien réel.

\section{Discussion : éléments d'analyse des tensions}

Les positions fondatrices des paradigmes précédents semblent absolument irréconciliables, peut-être parce que la distance épistémologique est trop grande entre eux pour qu'il puisse y avoir compromis.

D'un côté, on trouve la prétention de mesurer les effets de l'utilisation de méthodes et d'instruments, en séparant soigneusement les différentes variables entrant en jeu et en utilisant des procédures statistiques permettant dans l'idéal de prédire des performances. Ces procédures sont de deux types principaux :

- L'analyse de variance est un outil privilégié de l'expérimentation randomisée, parfois complétée par de l'analyse de covariance. Elle porte souvent sur des effectifs relativement modestes (de quelques dizaines à quelques centaines) et permet de comparer des moyennes entre groupes soumis à des conditions expérimentales contrastées.

- Les études corrélationnelles, comme on en trouve dans les études PISA, reposent, elles, sur de très grands effectifs et utilisent souvent des modèles linéaires, éventuellement hiérarchiques, 
permettant de mettre en évidence des co-variations de variables (en général « normalisées »), sans parvenir pour autant à atteindre à l'établissement de causalités.

De l'autre côté se trouvent des recherches se situant dans une perspective systémique ne cherchant pas à établir l'effet de la variation de variables contrôlées, mais visant à rendre intelligible des processus et à expliquer des situations en allant au-delà de mesures de l'influence de variables indépendantes sur des variables dépendantes et en prenant en compte des contextes élargis (Baron, 2019).

Il nous semble certain que l'opposition entre ces paradigmes ne se résume pas à des questions méthodologiques, mais est engagée par les choix conceptuels des chercheurs et renvoie in fine à l'asymétrie des relations entre ces derniers, les institutionnels et les praticiens, aux tensions entre les positions de recherche et d'expertise, de production de connaissance et d'intervention. Ces tensions sont constitutives de notre champ de recherche. Comme le rappelait Baron (2013), il s'est d'abord historiquement constitué comme un champ de pratiques avant d'engendrer un « champ de recherches organisé en un ensemble de petites communautés de chercheurs et d'innovateurs » (p. 2). Toute la recherche en éducation est concernée par la question de son « horizon praxéologique », pour reprendre la formule de Reuter (2007/2013), et la demande d'intervention, ne serait-ce que par la formation des enseignants, mais la demande sociale liée à des enjeux économiques et sociaux de l'instrumentation de l'éducation conduit à ce qu'Albero et Thibault (2009) ont caractérisé comme une « absence de distinction entre recherche et expertise » (p. 62).

Le paradigme prédictif entend répondre à une demande institutionnelle en mobilisant les instruments de mesure construits par les sciences humaines et sociales. Mais c'est justement parce que les questions émanent des institutions et décideurs que les termes mêmes du problème sont construits en dehors de la démarche scientifique. Ainsi, une bonne raison pour les chercheurs de se méfier des demandes d'expertises est que ces demandes n'émanent pas des connaissances scientifiques et des concepts consciemment construits dans un débat avec les données empiriques.

Les décideurs formulent des demandes dans leurs propres termes, non problématisés. C'est ainsi qu'en France le CSEN a affirmé que la science qui « a sa place en éducation » est celle qui fournit la « preuve » de « l'efficacité » « d'outils ». Aucun de ces termes n'est scientifiquement construit, la nature de ce qu'est une preuve n'est pas discutée (une pratique peut-elle constituer une preuve?), savoir si l'efficacité est une question pertinente non plus (voir Chaptal, 2009; Livingstone, 2012), ce qu'est un outil et s'il constitue une unité d'analyse adéquate encore moins.

En d'autres termes, si ce qui est mesuré relève du sens commun et non d'un travail de conceptualisation, la rupture épistémologique n’a pas lieu. 


\section{Conclusion : dépasser les oppositions pour faire (ré)émerger d'autres modèles de recherche}

Un consensus semble s'être installé sur le fait qu'il est intéressant de considérer des recherches participatives prenant en compte les contextes sociaux et développant une approche critique de l'usage des instruments (Bru, 2019, Collin et al., 2016; Laferrière et al., 2017; Laferrière, 2020).

En 2018, Martinand soulignait les difficultés des approches participatives, parmi lesquelles « l'élaboration d'une problématique de recherche commune, supposant habituellement de nouvelles conceptualisations », la reconnaissance réciproque des différents acteurs, les intérêts et agendas différents ainsi que « l'émergence et l'entretien de milieux médiateurs entre les acteurs de recherches et des "utilisateurs" potentiels ».

Comment avancer? Nous croyons nécessaire de discuter à nouveaux frais la tension entre prédiction, compréhension et explication, au regard des finalités d'intervention de la recherche dans le domaine de la technologie éducative afin de rapprocher chercheurs et praticiens sur le plan de l'invention instrumentale et de l'analyse des pratiques, mais en renforçant la rupture épistémologique entre les concepts des praticiens et ceux des chercheurs. Vaste et ambitieux programme!

Nous avons conscience de la tension qui existe entre des finalités aussi différentes que confirmer, prédire, expliquer, transformer... La recherche-action est sans doute l'approche la plus ancienne en éducation. En 2013, Pourtois et al. remarquaient qu'elle est « une action politique en quête de savoir » (p. 15) et que

être en recherche-action, c'est accepter de toujours se situer dans un entre-deux, de naviguer entre deux pôles opposés, c'est-à-dire de s'inscrire dans la dialectique, dans le paradoxe d'être [...] à la fois dans la confrontation et la reliance, dans la théorie et la pratique, dans la prévision et la prédiction, dans la problématologie et le résolutoire, dans la continuité et la rupture, dans le consensus et le dissensus, dans l'ouverture et la fermeture, dans la transparence. (p. 33)

Le plus difficile est bien sûr de gérer les contractions entre ces différents termes. Comment y parvenir? Dans un ordre d'idées similaire, Engeström (2007) nous propose une approche pratique, celle des laboratoires de changement, fondés théoriquement sur l'idée de double stimulation de Vygotsky, et qui vise à développer l'agentivité des groupes d'acteurs (Morselli \& Sannino, 2021). On est alors dans des démarches d'intervention d'inspiration systémique dont chacune apporte des éléments de compréhension de ce qui se joue et des pistes d'action mais qui ne convergent que modérément.

Cela suppose la création dans la durée de forums d'échange pluridisciplinaires et pluriculturels, tels que l'EDUsummIT afin de consolider des réseaux de communautés plurielles ayant intérêt à échanger afin de former des collectifs hybrides durables. Leur liant le plus solide sera sans doute le partage d'un ensemble d'idées et de valeurs militantes, mais celles-ci viennent toujours facilement en conflit avec les exigences de problématisation, c'est-à-dire procédant d'une rupture épistémologique, d'une construction conceptuelle contrôlée, discutant de la portée et du domaine de validité des résultats. Mais le jeu en vaut la chandelle... 


\section{Références}

Albero, B., \& Thibault, F. (2009). La recherche française en sciences humaines et sociales sur les technologies en éducation. Revue française de pédagogie, 169. http://rfp.revues.org/1434

Alter, N. (2000). L'innovation ordinaire. Paris : Presses Universitaires de France.

Bachelard, G. (1953). Le matérialisme rationnel. Paris : Presses Universitaires de France.

Bachelard, G. (1953). Le matérialisme rationnel. PUF.

Baron, G.-L. (2013). La recherche francophone sur les « technologies » en éducation : Réflexions rétrospectives et prospectives. STICEF - Sciences et technologies de l'information et de la communication pour l'éducation et la formation, 20. http://sticef.univlemans.fr/num/vol2013/16-baron-reiah/sticef 2013 NS baron 16.htm

Baron, G.-L., \& Bruillard, E. (1996). L’informatique et ses usagers dans l'éducation. Paris : Presses Universitaires de France. http://www.stef.ens-cachan.fr/annur/bruillard/usag somr.htm

Baron, G.-L., \& Bruillard, É. (2004). Quelques réflexions autour des phénomènes de scolarisation des technologies. Dans L.-O. Pochon et A. Maréchal (dir.), Entre technique et pédagogie. La création de contenus multimédias pour l'enseignement et la formation (p. 154-162). Neuchâtel : IRDP. http://mutatice.net/glbaron/lib/exe/fetch.php/2004 glb eb neuchatel.pdf.

Baron, G.-L., \& Depover, C. (dir.). (2019). Les effets du numérique sur l'éducation. Regards sur une saga contemporaine. Villeneuve-d'Ascq : Presses Universitaires du Septentrion.

Bart, D., \& Daunay, B. (2016). Les blagues à PISA. Vulaines-sur-Seine : Les éditions du Croquant.

Bernard, F.-X., \& Fluckiger, C. (2019). Innovation technologique, innovation pédagogique : éclairage de recherches empiriques en science de l'éducation. Spirale. Revue de recherches en éducation, 63(1), 3-10. https://www.cairn.info/revue-spirale-revue-de-recherches-en-education-2019-1.htm

Bernard, R. M. (2014). Things I have learned about meta-analysis since 1990 : Reducing bias in search of « the big picture »/Ce que j'ai appris sur la méta-analyse depuis 1990 : réduire les partis pris en quête d'une vue d'ensemble. Canadian Journal of Learning and Technology, 40(3). https://doi.org/10.21432/T2MW29

Béziat, J. (2003). Technologies informatiques à l'école primaire. De la modernité réformatrice à l'intégration pédagogique innovante. Contribution à l'étude des modes d'inflexion, de soutien, d'accompagnement de l'innovation [Thèse de doctorat en sciences de l'éducation, sous la direction de G-L Baron, Université Paris Descartes]. https://tel.archives-ouvertes.fr/tel$\underline{00437088 / \text { document }}$ 
Bianco, M. (2018). La réponse à des questions cruciales en éducation réside-t-elle dans un changement de paradigme? Éducation et didactique, 12(1), 121-128. https://journals.openedition.org/educationdidactique/3111

Bru, M. (2019). De quelques reconfigurations du rapport des recherches aux pratiques enseignantes. Les sciences de l'éducation. Pour l'ère nouvelle, 52(1), 79-101.

Bryk, A. S. (2017). Accélérer la manière dont nous apprenons à améliorer, Éducation et didactique, 11(2), 11-29. http://journals.openedition.org/educationdidactique/2796.

Chaptal, A. (2009). Mémoire sur la situation des TICE et quelques tendances internationales d'évolution. Sticef, 16. https:/www.persee.fr/doc/stice 1952-8302 2009 num 161993

Coburn, C. E. (2003). Rethinking scale: Moving beyond numbers to deep and lasting change. Educational Researcher, 32(6), 3-12. https://doi.org/10.3102/0013189X032006003

Collin, S. (2016). Le numérique en éducation : au-delà de l'impact. Diversité, 185, 137-142.

Collin, S., Brotcorne, P., Fluckiger, C., Grassin, J.-F., Guichen, N., Muller, C., Ntébutsé, J.-G., Ollivier, C., Roland, N., Schneider, É., \& Soubrié, T. (2016). Vers une approche sociocritique du numérique en éducation : une structuration à l'œuvre. Adjectif.net.

http://www.adjectif.net/spip/spip.php?article387

Cox, M. (2018). Researching information technology in education : Meeting the challenges of an everchanging environment. Dans J. Voogt, G. Knezek, R. Christensen et K.-W. Lai (dir.), Second Handbook of Information Technology in Primary and Secondary Education (p. 1103-1122). Cham: Springer.

Cuban, L. (1986). Teachers and machines. The classroom use of technology since 1920. New York: Teachers College/Columbia University Press.

Cukurova, M., \& Luckin, R. (2018). Measuring the impact of emerging technologies in education: A pragmatic approach. Dans J. Voogt, G. Knezek, R. Christensen et K.-W. Lai (dir.), Second Handbook of Information Technology in Primary and Secondary Education (p. 1179-1197). Cham: Springer.

De Munck, J. (2011). Les trois dimensions de la sociologie critique, SociologieS. http://sociologies.revues.org/3576

Engeström, Y. (2007). Putting Vygotsky to work: The Change Laboratory as an application of double stimulation. Dans H. Daniels, M. Cole et J. V. Wertsch (dir.), Putting Vygotsky to work: The change laboratory as an application of double stimulation. Cambridge University Press. http://www.helsinki.fi/cradle/documents/Engestrom\%20Publ/Change\%20Laboratory\%202007.P DF 
Fluckiger, C. (2019). Numérique en formation : des mythes aux approches critiques. Éducation permanente, 219(2), 17-30.

Fluckiger, C. (2020a). Ressources et outils face à la COVID-19 : critique d'un texte du CSEN sur la recherche qui a « sa place » en éducation. Adjectif, 3 . http://www.adjectif.net/spip/spip.php?article541

Fluckiger, C. (2020b). Les usages effectifs du numérique en classe et dans les établissements scolaires. Paris: Cnesco.

Fluckiger, C., Boucher, S., \& Daunay, B. (2016). Le temps vécu. Discours d'enseignants disposant d'un TNI sur le temps de préparation des cours. Distances et médiations des savoirs, 16. http://journals.openedition.org/dms/1642

Glass, G. V. (1976). Primary, secondary, and meta-analysis of research. Educational Researcher, 5(10), 3-8.

http://www.dataschemata.com/uploads/7/4/8/7/7487334/glass 1976 primarysecondarymetaanalys is.pdf

Granjon, F. (2014). La critique est-elle soluble dans les sciences de l'information et de la communication? Dans É. George et F. Granjon (dir.), Critique, sciences sociales et communication (p. 291-355). Paris : Mare \& Martin.

Jouet, J. (2000). Retour critique sur la sociologie des usages. Réseaux, 18(100), 487-522.

Laferrière, T. (2020). La recherche en partenariat pour l'enseignement d'hier à demain. Revue hybride de l'éducation, 4(1), 94-115. https://doi.org/10.1522/rhe.v4i1.974

Laferrière, T., Montane, M., Gros, B., Alvarez, I., Bernaus, M., Breuleux, A., Allaire, S., Hamel, C., \& Lamon, M. (2010). Partnerships for knowledge building: An emerging model. Canadian Journal of Learning and Technology, 36(1). https://doi.org/10.21432/T2R59Z

Laferrière, T. (2017). Les défis de l'innovation selon la théorie de l'activité : Le cas de l'école (éloignée) en réseau. Canadian Journal of Education/Revue canadienne de l'éducation, 40(2), 1-30. https://journals.sfu.ca/cje/index.php/cje-rce/article/view/3110

Laferrière, T. (2020). La recherche en partenariat pour l'enseignement d'hier à demain. Revue hybride de l'éducation, 4(1), 94-115.

Livingstone, S. (2012). Critical reflections on the benefits of ICT in education. Oxford Review of Education, 38(1), 9-24. http://eprints.1se.ac.uk/42947/

Martinand, J.-L. (2002). Entretien d'Evelyne Burguière avec Jean-Louis Martinand. Recherche \& formation, 40(1), 87-94. https://doi.org/10.3406/refor.2002.1762 
Martinand, J.-L. (2018). Les produits des recherches participatives en sciences de l'éducation : Éléments pour leur prise en compte évaluative. Recherche participative et sciences de l'éducation. Hommage aux travaux de François Villemonteix. https://rech-part-sedu.sciencesconf.org/resource/page/id/7

Mialaret, G. (2016). Les origines et l'évolution des sciences de l'éducation en pays francophones. Les sciences de l'éducation. Pour l'ère nouvelle, 49(3), 53-69. https://www.cairn.info/revue-lessciences-de-1-education-pour-1-ere-nouvelle-2016-3-page-53.htm

Mœglin, P. (2012). Ardoises numériques, changement de paradigme? Communication présentée au colloque EcriTech3. http://www.ecrituretechnologie.com/wpcontent/uploads/2012/09/ecritech3 avril2012 transcription P MOEGLIN.p df

Morel, R. P., Coburn, C., Catterson, A. K., \& Higgs, J. (2019). The multiple meanings of scale: Implications for researchers and practitioners. Educational Researcher, 48(6), 369-377. https://doi.org/10.3102/0013189X19860531

Morselli, D., \& Sannino, A. (2021). Testing the model of double stimulation in a Change Laboratory. Teaching and Teacher Education, 97, 103224. https://doi.org/10.1016/j.tate.2020.103224

Nogry, S., \& Sort, C. (2016). Le temps de l'appropriation d'une classe mobile par les enseignants à l'école primaire. Distances et médiations des savoirs, 16.

http://journals.openedition.org/dms/1655

Pachler, N., \& Turvey, K. (2018). Looking back, moving forward: Impact and measurability of the use of educational technology. Dans J. Voogt, G. Knezek, R. Christensen et K.-W. Lai (dir.), Second Handbook of Information Technology in Primary and Secondary Education (p. 1199-1223). Cham: Springer.

Pogrow, S. (2017). The failure of the U.S. education research establishment to identify effective practices: Beware effective practices policies. Education Policy Analysis Archives, 25(5). https://doi.org/10.14507/epaa.25.2517

Pourtois, J.-P., Desmet, H., \& Humbeeck, B. (2013). La recherche-action, un instrument de compréhension et de changement du monde. Recherches qualitatives, 15, 25-35. https://doi.org/10.14507/epaa.25.2517

Reuter, Y. (2007/2013) Dictionnaire des concepts fondamentaux des didactiques, 3 éd. Bruxelles : De Boeck.

Saussez, F., \& Lessard, C. (2009). Entre orthodoxie et pluralisme, les enjeux de l'éducation basée sur la preuve. Revue française de pédagogie, 168. http://journals.openedition.org/rfp/1804 
Selwyn, N. (2010). Looking beyond learning: Notes towards the critical study of educational technology. Journal of Computer Assisted Learning, 26(1), 65-73. https://doi.org/10.1111/j.13652729.2009.00338.x

Villemonteix, F., \& Khaneboubi, M. (2013). Étude exploratoire sur l'utilisation d'iPads en milieu scolaire : entre séduction ergonomique et nécessités pédagogiques. Sticef, 20. http://sticef.univlemans.fr/num/vol2013/13-villemonteix-atame/Sticef_2013 NS_villemonteix_13.htm 


\section{Autours}

Georges-Louis Baron est professeur émérite de sciences de l'éducation à l'Université de Paris, laboratoire EDA. Il s'intéresse à l'ensemble des utilisations éducatives des technologies, depuis ce qui relève de la technologie éducative jusqu'à à la didactique de l'informatique, avec un intérêt particulier porté aux différents acteurs des innovations liées aux technologies. Courriel : Georges-louis.baron@uparis.fr

Cédric Fluckiger est professeur des Universités en sciences de l'éducation au laboratoire CIREL, Université de Lille. Il étudie la culture numérique des élèves et des étudiants; les contenus informatiques à l'école primaire et secondaire ; et porte un regard critique sur la technologie éducative face aux discours institutionnels et médiatiques. Courriel : cedric.fluckiger@univ-lille.fr

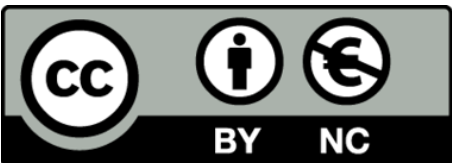

This work is licensed under a Creative Commons Attribution-NonCommercial CCBY-NC 4.0 International license. 